\title{
BodyMap: A Collection of 3' ESTs for Analysis of Human Gene Expression Information
}

\author{
Shoko Kawamoto, ${ }^{1}$ Junji Yoshii, ${ }^{2}$ Katsuya Mizuno, ${ }^{2}$ Kouichi Ito, ${ }^{1}$ \\ Yasuhide Miyamoto, ${ }^{1}$ Tadashi Ohnishi, ${ }^{1}$ Ryo Matoba, ${ }^{1}$ Naohiro Hori, ${ }^{1}$ \\ Yuhiko Matsumoto, ${ }^{1}$ Toshiyuki Okumura, ${ }^{1}$ Yuko Nakao, ${ }^{1}$ Hisae Yoshii, ${ }^{1}$ \\ Junko Arimoto, ${ }^{1}$ Hiroko Ohashi, ${ }^{1}$ Hiroko Nakanishi, ${ }^{1}$ Ikko Ohno, ${ }^{1}$ Jun Hashimoto, ${ }^{1}$ \\ Kota Shimizu, ${ }^{1}$ Kazuhisa Maeda, ${ }^{1}$ Hiroshi Kuriyama, ${ }^{1}$ Koji Nishida, ${ }^{1}$ \\ Akiyo Shimizu-Matsumoto, ${ }^{1}$ Wakako Adachi, ${ }^{1}$ Reiko Ito, ${ }^{1}$ Satoshi Kawasaki, ${ }^{1}$ \\ K.S. Chae, ${ }^{1}$ Katsuji Murakawa, ${ }^{1}$ Masahiro Yokoyama, ${ }^{1}$ Atsushi Fukushima, ${ }^{1}$ \\ Teruyoshi Hishiki, ${ }^{1}$ Akihiko Nakaya, ${ }^{3}$ Jun Sese, ${ }^{3}$ Norikazu Monma, ${ }^{3}$ \\ Hitoshi Nikaido, ${ }^{3}$ Shinichi Morishita, ${ }^{3}$ Kenichi Matsubara, ${ }^{4}$ and Kousaku Okubo ${ }^{5}$ \\ ${ }^{1}$ Institute for Molecular and Cellular Biology, Osaka University, Osaka 565-0871, Japan; ${ }^{2}$ Hitachi Software Engineering Co., \\ Ltd., Yokohama 231-0015, Japan; ${ }^{3}$ Department of Genome Knowledge Discovery System, Institute of Medical Science, \\ University of Tokyo, Tokyo 108-8639, Japan; ${ }^{4}$ Internal Institute for Advanced Study, Kyoto 619-0225, Japan
}

\begin{abstract}
BodyMap is a collection of site-directed 3' expressed sequence tags (ESTs) (gene signatures, GSs) that contains the transcript compositions of various human tissues and was the first systematic effort to acquire gene expression data. For the construction of BodyMap, cDNA libraries were made, preserving abundance information and histologic resolutions of tissue mRNAs. By sequencing 164,000 randomly selected clones, 88,587 GSs that represent chromosomally coded transcripts have been collected from 51 human organs and tissues. They were clustered into 18,722 independent $3^{\prime}$ termini from transcripts, and more than 3000 of these were not found among ESTs assembled in UniGene (Build 75). Assessment of the prevalence of polyadenylation signals and comparison with GenBank cDNAs indicated that there was no significant contamination by internally primed cDNAs or genomic fragments but that there was a relatively high incidence (12\%) of alternative polyadenylation sites. We evaluated the sensitivity and resolution of expression information in BodyMap by in silico Northern hybridization and selection of tissue-specific gene probes. BodyMap is a unique resource for estimation of the absolute abundance of transcripts and selection of gene probes for efficient hybridization-based gene expression profiling. [BodyMap data are available at http://bodymap.ims.u-tokyo.ac.jp.]
\end{abstract}

In the early phase of its development, the expressed sequence tag (EST) collection (Adams et al. 1993, 1995) primarily served as a catalog to be screened for clones of interest by sequence homology. In the next phase, gene coverage was pursued (Aaronson et al. 1996; Williamson 1999) by using normalized libraries and/ or highly complex sources (Soares et al. 1994; Hillier et al. 1996) to use the entries as markers to create a transcript map of the human genome, after clustering redundantly accumulated ESTs into gene units (Schuler et al. 1996). As genome sequencing efforts progress, ESTs have been used for exon identification (Dunham et al. 1999; Hattori et al. 2000), and they are being mapped and organized in the framework of genome

\section{${ }^{5}$ Corresponding author.}

E-MAIL kousaku@imcb.osaka-u.ac.jp; FAX 81-6-6877-1922.

Article and publication are at www.genome.org/cgi/doi/10.1101/ gr.151500. sequence at a resolution of single nucleotides. Progress in the integration of ESTs into the genomic sequence will make EST data more of an expression of gene records rather than merely a pool of nucleotide sequences. Reflecting this trend, the major EST collection projects have shifted emphasis from efficiency of identifying novel sequences to meaningful source selection, such as coverage of a majority of cancer types (Strausberg et al. 2000).

BodyMap is a collection of site-directed 3' ESTs (gene signatures, GSs) designed as an anatomical database of human gene expression in which sequences are used as identifiers (Okubo et al. 1992). Construction of BodyMap began in 1991 (Okubo et al. 1991) and representative human tissues and organs have been incorporated. During the collection of GSs, nonstructural information about the mRNA, including transcript abundance and anatomical distribution, was pre- 
served. The libraries were constructed from wellcharacterized sources by using methods that minimize the differences in cloning efficiencies among transcripts, and libraries were never amplified before sequencing (Okubo et al. 1991). Accordingly, BodyMap has characteristics distinct from those of other public EST data sets, which were generated as sequence collections at the expense of expression information (Bonaldo et al. 1996). BodyMap has been used in the isolation and characterization of tissue-specific transcripts (Nishida et al 1996; Ohno et al. 1996; Maeda et al. 1997; Shimizu-Matsumoto et al. 1997) and in disease gene identification (Irvine et al. 1997; Nishida et al. 1997). Here we describe the structure and features of 88,587 GSs from human tissues collected in BodyMap.

\section{RESULTS}

\section{Sources and Library Construction}

The numbers of informative 3' site-directed ESTs representing chromosomally coded genes are summarized in Table 1. We refer to these 3' ESTs, covering restricted 3 ' ends in the sense direction, as gene signatures (GSs) (Okubo et al. 1992). Sources were selected to cover the most representative tissues and cell types. Emphasis was placed on pure connective tissues and epithelial cells, which are underrepresented in dbEST. In every case, tissue preparation was performed carefully, sometimes by microscopy, to minimize contamination by other cell types. For example, human epithelial cells were prepared by careful isolation of a monolayer or layers of cells free from visible contamination by connective tissues and blood cells (Ohnishi et al. 1999). As a result, for example, the sequence of the immunoglobulin $\lambda$ chain transcript, which was found in $1 \%$ $(11 / 870)$ of clones from colonic mucosa having a thin lining of loose connective tissue (lamina propria), was not identified in 20,440 clones from purified epithelium. Because of the elaborate manipulation steps, libraries were sometimes constructed by direct priming of less than a microgram of total RNA. Nevertheless, contamination by ribosomal RNA was very low $(0.26 \%)$, probably because of the high specificity of first-strand synthesis with a low concentration vector primer.

\section{Validation of Collected GSs}

Collected GS sequences were evaluated if they represented true mRNA termini. Of 3928 independent GS sequences that matched GenBank entries, 3470 (88\%) represented the most 3' MboI fragments of the deposited cDNA sequences. The rest represented alternatively polyadenylated mRNAs or internally primed artifacts that cannot be discriminated by sequence inspection of individual cases. Thus, the presence of the
poly(A) addition signals upstream of the addition site was used for the validation as mass data. Canonical signal (AATAAA) and sequences with single-base substitutions were examined $10 \mathrm{bp}$ to $50 \mathrm{bp}$ from the poly(A) tail in 4431 independent GS sequences (Fig. 1A). 1 In $93 \%$ of GS sequences, AATAAA or a single base variant was found. The prevalence of AATAAA and single base variants was quite similar to that observed in 1123 GenBank human cDNAs with clear annotations of poly(A) sites. In the case of 3' ESTs deposited in dbEST, the proportions of signals differed greatly between those starting with a stretch of Ts and those without them (Fig. 1A). The former has very similar signal occurrence, but in the latter the proportion of AATAAA is greatly reduced, indicating that the short region following a poly(T) stretch was trimmed before data submission as reported (Hillier et al. 1996). The 458 BodyMap GS that matched internal regions of GenBank mRNAs had similar frequencies of hexanucleotide signals, suggesting that the majority of them are also polyadenylated in vivo. Because we counted not only the well-known single-base variants, such as ATTAAA, but also all of the possible single-base substitutions, the fraction of each of these variants was compared for cDNA ends having only one candidate signal (Fig. 1B). The proportion was consistent across GenBank primate sequences, BodyMap, and untrimmed 3' ESTs. Trimmed 3' ESTs served as nonterminal controls. This agreement between the three sets of data suggests that some of the uncommon hexanucleotide variants, such as BATAAA and $\operatorname{AATABA}(B=T, G, C)$, are functional and that most of the cDNA sequences with only single-base variants in either set of data represent true $3^{\prime}$ termini of transcripts.

\section{Constitution of GS Population}

Without exception, the most recurrent GSs in differentiated cells or in adult tissues were from nonhousekeeping genes (Table 1). Some were unique to each tissue, and some were shared among cells of the same lineage. The fraction of the most abundant GS varied more than tenfold across tissues or cell types. There were six tissues in which more than $10 \%$ of the total ESTs were attributable to a single GS cluster (Fig. 2). They were secretory epithelia or muscular tissues. In the remaining tissues, the content varied by a small percent (mean, $2.5 \%$; SD, $1.8 \%$ ).

The characteristics of the GS population for each source group-nervous tissues, connective tissues, and epithelial tissues-are illustrated in the accumulated frequency curve (Fig. 3) in which the cumulative sums of occurrences were plotted in descending order of GS occurrence. The epithelial and connective tissues have very similar curves, whereas that of the nervous system is clearly shifted downward. The curve for neural tis- 


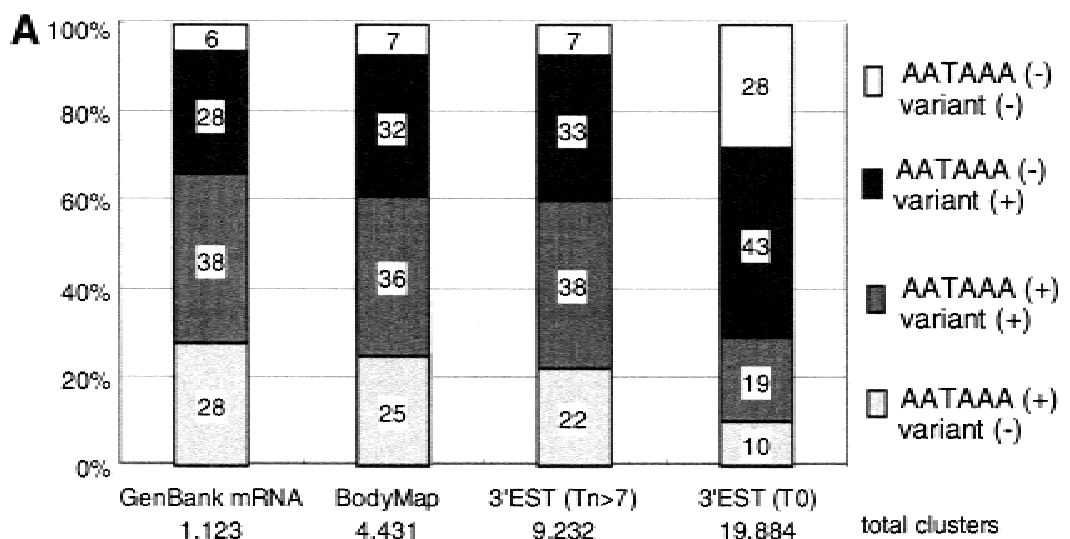

B

B Frequencies in $\%$ among AATAAA $(-)$ variant $(+)$

\begin{tabular}{|c|c|c|c|c|c|}
\hline & GenBank mRNA & BodyMap GS & $3^{\prime} \mathrm{EST}(\mathrm{Tn}>7)$ & 3'EST (To) & $\begin{array}{l}\text { In vitro activity } \\
\text { \{relative to AATAAA in } \%\end{array}$ \\
\hline \multirow{2}{*}{\multicolumn{6}{|c|}{$\begin{array}{l}\text { TATAAA } \\
\text { GATAAA }\end{array}$}} \\
\hline & & 14 & & & \\
\hline CATAAA & 믕 8 & 田 5 & $\mathbf{E}_{5}$ & 5 & 18 \\
\hline ATTAAA & & & 달 & I 14 & \\
\hline AGTAAA & 8 & 14 & $\mathbb{1}_{11}$ & & 29 \\
\hline ACTAAA I & & 12 & 13 & 4 & $\mathbf{D}_{11}$ \\
\hline AAAAAA & & 0 & $I_{1}$ & 13 & 15 \\
\hline AAGAAA I & & 12 & 3 & 9 & I 6 \\
\hline AACAAA & & 12 & | 2 & E 6 & 14 \\
\hline AATTAA & & l1 & 0 & $\mathbf{I} 3$ & I 2 \\
\hline AATGAA I & & 12 & 13 & 7 & $I_{4}$ \\
\hline AATCAA & & |1 & 0 & 13 & I 4 \\
\hline AATATA & $\mathbf{D}$ & 5 & 国 & E6 & $\mathbf{D}_{10}$ \\
\hline AATAGA & & | 2 & 11 & 13 & I3 \\
\hline AATACA & 7 & E6 & 5 & 5 & $\mathbf{D}_{11}$ \\
\hline AATAAT I & & |1 & $I_{2}$ & $\mathbf{D}_{6}$ & I5 \\
\hline AATAAG & & 0 & $I_{1}$ & $\mathbf{1} 4$ & 12 \\
\hline AATAAC I & 1 & I & $I_{1}$ & 13 & 14 \\
\hline
\end{tabular}

Figure 1 Distribution of AATAAA and single-base variants in $3^{\prime}$ ends of ESTs. (A) The hexanucleotide signals from the $3^{\prime}$ ends of four sets of cDNA sequences. The regions $10-50$ bp from the polyadenylation sites were examined for the presence of AATAAA or its singlebase variants. The $3^{\prime}$ ESTs from dbEST were divided into those starting with more than seven Ts $(T n>7)$ and those without a $T$ in the first position $\left(T_{0}\right)$. (B) The prevalence of each single-base variant in the CDNA termini with only one variant signal and no AATAAA within the 10-50-bp region from the poly(A) tail in each of four data sets is shown. The frequencies (\%) of all 18 possible variants in each data set are shown beside bars. In vitro polyadenylation activities for each variant measured in the context of the SV40 polyadenylation signal are reproduced from the literature (Wickens et al. 1984; Sheets et al. 1990)

sues did not overlap with the others at a credit level of 0.85 in the top 486 genes. As seen in Figure 2, 50\% of the mass is accounted for by $\sim 500$ genes in connective and epithelial tissues but by $>900$ genes in nervous tissue.

\section{Overlapping with dbEST}

To further characterize BodyMap data, we compared them with dbEST entries in UniGene (Build 75) that were clustered into 72,831 physical and annotational clusters. Of 18,722 GS clusters composed of 89,831 GS tags in BodyMap, 3,382 GS clusters did not match ESTs listed.

The GS in overlapping fraction have an average redundancy of 5.6 in BodyMap, whereas it was 1.3 in the GS cluster unique to BodyMap. GS unique to BodyMap were distributed at frequencies of $1 \%-5 \%$ in every library (mean, $4.0 \%$; SD, $2.2 \%$ ) and had hexanucleotide signal occurrences similar to the rest (data not shown). Nervous system tissues had a slightly greater content of unique GS (mean, 5.4\%; SD, 2.1\%) than was found in other tissues (mean, $3.8 \%$; SD, $1.8 \%$ ). These values equaled or exceeded $10 \%$ in only two libraries: full thickness of skin $(10 \%)$ and fetal neuron (11\%).

\section{In Silico RNA Experiments}

The primary goal for the construction of BodyMap was to create a genes $x$ tissues matrix of transcription level that could be used for in silico experiments such as Northern hybridization and subtraction cloning. Although the depth of the clone collection limits the sensitivity and specificity of experiments, for abundant clones these primary objectives have been achieved. The sensitivity of the present matrix was assessed by probing the data with several genes known to have moderate expression levels and known tissue specificities. As shown in Table 2, the distributions of cytoskeletal intermediate filaments and collagens suggest that this clear segregation is applicable also to anonymous genes with similar expression levels. Such pure segregation patterns are not seen in libraries constructed from complex starting materials.

Another example of an in silico experiment is selection of genes with given patterns of expression. For example, genes differentially expressed in myeloid cells, based on the criteria that frequency variation between myeloid cells and nonmyeloid cells was highly significant $(P<.005)$, are shown in Table 3 . By increas- 
Kawamoto et al.

Table 1. The Most Abundant Transcripts in Human Tissues

\begin{tabular}{|c|c|c|c|c|c|c|c|c|c|c|c|}
\hline B01.hl60 & 857 & & $\begin{array}{l}\text { C08.Aortic } \\
\text { media }\end{array}$ & 1002 & & N01. Retina & 877 & & $\begin{array}{c}\text { X01. } \\
\text { hepG2 }\end{array}$ & 740 & \\
\hline $\begin{array}{l}\text { Ribosomal } \\
\text { protein S8 }\end{array}$ & X67247 & 24 & Elastin & M17282 & 155 & Opsin & K02281 & 14 & $\mathrm{EF}-1 \alpha$ & X16869 & 17 \\
\hline $\begin{array}{l}\text { Ribosomal } \\
\text { protein L9 }\end{array}$ & U09953 & 22 & Osteonectin & J03040 & 27 & $\begin{array}{l}\mathrm{Na} / \mathrm{K} \\
\quad \text { ATPase } \beta 2\end{array}$ & D87330 & 13 & Albumin & L00133 & 17 \\
\hline $\begin{array}{l}\text { Ribosomal } \\
\text { protein L23 }\end{array}$ & X53777 & 17 & $\begin{array}{l}\text { Ribosomal } \\
\text { protein } \\
\text { L21 }\end{array}$ & X89401 & 16 & Aldolase C & X07292 & 10 & TPT-1 & X16064 & 9 \\
\hline $\begin{array}{l}\text { Ribosomal } \\
\text { protein L7a }\end{array}$ & X52138 & 16 & GS13325 & None & 16 & $\begin{array}{l}\text { Ribosomal } \\
\text { protein L9 }\end{array}$ & U09953 & 9 & $\begin{array}{l}\text { Ribosomal } \\
\text { protein L31 }\end{array}$ & X69181 & 9 \\
\hline $\begin{array}{l}\text { B02. hl60/ } \\
\text { DMSO }\end{array}$ & 1081 & & $\begin{array}{l}\text { C09. } \\
\text { Ventricle } \\
\text { muscle }\end{array}$ & 3785 & & N02. Cortex & 2242 & & $\begin{array}{l}\text { X02. } \\
\text { Neonate } \\
\text { liver }\end{array}$ & 739 & \\
\hline$\beta$-actin & X00351 & 14 & $\begin{array}{l}\text { Myosin } \\
\text { heavy } \\
\text { chain }\end{array}$ & M25139 & 101 & $\begin{array}{l}\text { Myelin basic } \\
\text { protein }\end{array}$ & M13577 & 49 & Albumin & L00133 & 227 \\
\hline $\begin{array}{l}\text { HHCPA78 } \\
\text { homolog }\end{array}$ & S73591 & 6 & $\begin{array}{l}\text { lg- } \lambda \text { light } \\
\text { chain }\end{array}$ & D01059 & 75 & hng/RC3 & Y15059 & 14 & $\begin{array}{l}\text { Apolipo- } \\
\text { protein B }\end{array}$ & J02775 & 38 \\
\hline $\begin{array}{l}\text { Ribosomal } \\
\text { protein L3 }\end{array}$ & M90054 & 5 & Myoglobin & X00373 & 60 & $\begin{array}{l}\text { Apolipo- } \\
\text { protein J }\end{array}$ & M74816 & 13 & $\begin{array}{l}\alpha 2 \text {-HS-glyco- } \\
\text { protein }\end{array}$ & M16961 & 21 \\
\hline L-plastin & L05492 & 5 & $\begin{array}{c}\text { Troponin C, } \\
\text { skel/card }\end{array}$ & M37984 & 53 & Aldolase C & X07292 & 9 & $\begin{array}{l}\text { Haptoglobin } \\
\alpha 1 S\end{array}$ & X00637 & 16 \\
\hline B03. hl60/TPA & 889 & & $\begin{array}{l}\text { C10. atrial } \\
\text { muscle }\end{array}$ & 2823 & & $\begin{array}{l}\text { N03. } \\
\text { cerebellum }\end{array}$ & 1107 & & $\begin{array}{l}\text { X03. fetal } \\
\text { liver }\end{array}$ & 641 & \\
\hline $\mathrm{EF}-1 \alpha$ & X16869 & 26 & ANF & M54951 & 203 & GFAP & S40719 & 22 & Albumin & L00133 & 109 \\
\hline $\begin{array}{l}\text { Methionine } \\
\text { AT-a }\end{array}$ & L43509 & 14 & $\begin{array}{l}\text { Actin, } \\
\quad \text { a-cardiac }\end{array}$ & J00073 & 88 & Aldolase C & X07292 & 7 & $\begin{array}{l}\text { Haptoglobin } \\
\quad \alpha 1 S\end{array}$ & X00637 & 27 \\
\hline Ferritin L & M11147 & 14 & $\alpha$ B-crystallin & S45630 & 27 & $\begin{array}{l}\text { Myelin basic } \\
\text { protein }\end{array}$ & M13577 & 5 & $\gamma$-G globin & X55656 & 17 \\
\hline TPT-1 & X16064 & 13 & $\begin{array}{l}\text { Troponin T, } \\
\text { cardiac }\end{array}$ & X74819 & 25 & Apolipoprotein & M74816 & 5 & $\begin{array}{l}\text { Apolipo- } \\
\text { protein All }\end{array}$ & X04898 & 14 \\
\hline $\begin{array}{l}\text { B04. granulo- } \\
\text { cyte }\end{array}$ & 1164 & & $\begin{array}{l}\text { C11. } \\
\text { Skeletal } \\
\text { muscle }\end{array}$ & 4527 & & $\begin{array}{l}\text { N04. } \\
\text { Neuroblast }\end{array}$ & 1235 & & $\begin{array}{l}\text { X04. Adult } \\
\text { liver }\end{array}$ & 956 & \\
\hline $\begin{array}{l}\beta \text {-2-micro- } \\
\text { globulin }\end{array}$ & M17987 & 25 & $\begin{array}{l}\alpha \text {-actin, } \\
\text { skeletal }\end{array}$ & M20543 & 301 & $\mathrm{EF}-1 \alpha$ & X16869 & 19 & Albumin & L00133 & 279 \\
\hline $\begin{array}{l}\text { Spermidine/ } \\
\text { spermineAT }\end{array}$ & M77693 & 22 & $\begin{array}{l}\text { Myosin } \\
\text { heavy } \\
\text { chain }\end{array}$ & X03741 & 173 & H3.3 histone & M11354 & 17 & $\begin{array}{l}\text { Haptoglobin } \\
\quad \alpha 1 \mathrm{~S}\end{array}$ & X00637 & 41 \\
\hline HLA-Cw1 & M26429 & 21 & $\begin{array}{l}\text { Myosin } \\
\text { heavy } \\
\text { chain }\end{array}$ & X03740 & 137 & $\begin{array}{l}\text { ribosomal } \\
\text { protein L9 }\end{array}$ & U09953 & 16 & $\alpha-1$ acid gp & M13692 & 20 \\
\hline $\begin{array}{l}\text { Pre-B enhancing } \\
\text { factor }\end{array}$ & U02020 & 20 & $\begin{array}{c}\text { Troponin C, } \\
\text { skeletal }\end{array}$ & X07898 & 121 & TPT-1 & X16064 & 15 & $\begin{array}{l}\text { Apolipo- } \\
\text { protein B }\end{array}$ & J02775 & 19 \\
\hline B05. CD8 T cell & 1104 & & $\begin{array}{l}\text { C12. Hair } \\
\text { follicle }\end{array}$ & 2164 & & $\begin{array}{l}\text { N05. } \\
\text { Caudate } \\
\text { nucl. }\end{array}$ & 1077 & & X05. Lung & 874 & \\
\hline $\begin{array}{c}\beta \text {-2-micro- } \\
\text { globulin }\end{array}$ & M17987 & 16 & Fibronectin & K00799 & 84 & hng/RC3 & Y15059 & 12 & $\begin{array}{l}\text { Pulmonary } \\
\text { SAP }\end{array}$ & M30838 & 87 \\
\hline TPT-1 & X16064 & 12 & COL1A1 & M32798 & 57 & TALLA-1 & D29808 & 11 & $\begin{array}{l}\text { Clara cells } 10 \\
\text { kd prot. }\end{array}$ & U01101 & 31 \\
\hline EF-1a & X16869 & 10 & $\mathrm{EF}-1 \alpha$ & X16869 & 43 & $\begin{array}{l}\text { Myelin basic } \\
\text { protein }\end{array}$ & M13577 & 8 & $\begin{array}{l}\text { HLA-E heavy } \\
\text { chain }\end{array}$ & X64881 & 12 \\
\hline $\begin{array}{l}\text { Yeast rp L4 } \\
\text { homolog }\end{array}$ & Z12962 & 10 & Osteonectin & J03040 & 30 & KIAA0607 & AB011179 & 7 & Fibronectin & K00799 & 10 \\
\hline B06. CD4 T cell & 1028 & & $\begin{array}{l}\text { E1. } \\
\text { Keratino- } \\
\text { cyte }\end{array}$ & 820 & & $\begin{array}{l}\text { N06. } \\
\text { Thalamus }\end{array}$ & 912 & & $\begin{array}{l}\text { X06. Colon } \\
\text { mucosa }\end{array}$ & 921 & \\
\hline $\begin{array}{l}\beta \text {-2-micro- } \\
\text { globulin }\end{array}$ & M17987 & 19 & $\begin{array}{l}\text { Cytokeratin } \\
14\end{array}$ & J00124 & 15 & $\begin{array}{l}\text { Myelin basic } \\
\text { protein }\end{array}$ & M13577 & 36 & L-FABP & M10617 & 40 \\
\hline $\begin{array}{l}\text { Ribosomal } \\
\text { protein L11 }\end{array}$ & X79234 & 14 & $\begin{array}{l}\text { Metallothio- } \\
\text { nein }\end{array}$ & V00594 & 10 & GFAP & S40719 & 8 & Galectin-4 & AF01483 & 18 \\
\hline TPT-1 & X16064 & 13 & Lipocortin II & DOC & 9 & & M74 & 7 & CLC & AF03940 & 13 \\
\hline $\begin{array}{l}23 \mathrm{kD} \text { highly } \\
\text { basic protein }\end{array}$ & X56932 & 12 & $\begin{array}{l}\text { Ribosomal } \\
\text { protein } \\
\text { S19 }\end{array}$ & M81757 & 8 & Sox 8 & AF164104 & 6 & $\begin{array}{l}\text { lg- } \lambda \text {-light } \\
\text { chain }\end{array}$ & D01059 & 12 \\
\hline
\end{tabular}


Table 1. (Continued)

\begin{tabular}{|c|c|c|c|c|c|c|c|c|c|c|c|}
\hline $\begin{array}{l}\text { C01. Adipose } \\
\text { tissue }\end{array}$ & 1488 & & E02. Cornea & 2793 & & $\begin{array}{l}\text { N07. } \\
\text { Putamen }\end{array}$ & 871 & & $\begin{array}{l}\text { X07. } \\
\text { Small } \\
\text { cell ca. }\end{array}$ & 843 & \\
\hline Gelatin BP & $\mathrm{AB} 012165$ & 19 & $\begin{array}{l}\text { Apolipo- } \\
\text { protein J }\end{array}$ & M74816 & 73 & $\begin{array}{l}\text { Myelin } \\
\text { basic } \\
\text { protein }\end{array}$ & M13577 & 8 & $\underset{B B C 1}{\text { lung }}$ & X64707 & 8 \\
\hline $\begin{array}{l}\text { Ribosomal } \\
\text { protein S8 }\end{array}$ & X67247 & 16 & $\begin{array}{l}\text { Cytokeratin } \\
12\end{array}$ & D78367 & 55 & GS04506 & None & 8 & $\begin{array}{c}23 \text { kD } \\
\text { highly } \\
\text { basic } \\
\text { prot. }\end{array}$ & X56932 & 7 \\
\hline apM2 & D45370 & 15 & apM2 & NM006829 & 40 & TPT-1 & X16064 & 7 & $\begin{array}{l}\text { Ribosomal } \\
\text { protein } \\
\text { S11 }\end{array}$ & X06617 & 7 \\
\hline TPT-1 & $\times 16064$ & 14 & Ferritin $\mathrm{H}$ & M11146 & 31 & $\begin{array}{l}\mathrm{Na} / \mathrm{K} \\
\text { ATPase } \\
\beta 2\end{array}$ & D87330 & 7 & $\begin{array}{c}\text { Ribosomal } \\
\text { protein } \\
\text { L7a }\end{array}$ & $\times 52138$ & 6 \\
\hline Fibronectin & K00799 & 36 & $\begin{array}{c}\beta \text {-2-micro- } \\
\text { globulin }\end{array}$ & M17987 & 23 & $\mathrm{EF}-1 \alpha$ & X16869 & 26 & COL3A1 & X14420 & 20 \\
\hline ТРТ-1 & $\times 16064$ & 14 & $\begin{array}{l}\text { Cytokeratin } \\
13\end{array}$ & $\times 52426$ & 23 & $\begin{array}{c}\text { Ribosomal } \\
\text { protein } \\
\text { S17 }\end{array}$ & M13932 & 14 & $\begin{array}{c}\text { Thymosin } \\
\beta 4\end{array}$ & M17733 & 15 \\
\hline PAI-1 & $\times 13345$ & 12 & Lipocortin & X05908 & 8 & GFAP & S40719 & 12 & $\begin{array}{l}\text { lg- } \lambda \text { light } \\
\text { chain }\end{array}$ & D01059 & 14 \\
\hline CTGF & X78947 & 12 & EF-4All & D30655 & 8 & $\begin{array}{l}\text { Thymosin } \\
\text { } 34\end{array}$ & M17733 & 10 & $\begin{array}{l}\text { lg-к light } \\
\text { chain }\end{array}$ & M11937 & 13 \\
\hline $\begin{array}{l}\text { C03. Osteo- } \\
\text { blast* }^{*}\end{array}$ & 928 & & $\begin{array}{l}\text { E04. Intest. } \\
\text { Metaplasia }\end{array}$ & 2192 & & $\begin{array}{l}\text { N09. } \\
\text { Schwann } \\
\text { cell* }\end{array}$ & 975 & & $\begin{array}{l}\text { X09. } \\
\text { Squamou } \\
\text { cell ca. } \\
\text { lung }\end{array}$ & 1190 & \\
\hline COL1A2 & J03464 & 25 & Calcyclin & J02763 & 25 & $\begin{array}{l}\text { Ribosomal } \\
\text { protein } \\
\text { L10 }\end{array}$ & $A B 007170$ & 18 & Calcyclin & J02763 & 23 \\
\hline Fibronectin & K00799 & 22 & $\mathrm{EF}-1 \alpha$ & $\times 16869$ & 20 & $\begin{array}{l}\text { Ribosomal } \\
\text { protein } \\
\text { L9 }\end{array}$ & U09953 & 17 & $\begin{array}{l}\text { Ferritin L } \\
\text { chain }\end{array}$ & M11147 & 14 \\
\hline Osteonectin & 103040 & 20 & $\begin{array}{l}\text { Amino- } \\
\text { peptidase } \\
\mathrm{N}\end{array}$ & M22324 & 17 & $\begin{array}{l}\text { Ribosomal } \\
\text { protein } \\
\text { S19 }\end{array}$ & M81757 & 17 & Cystatin B & L03558 & 11 \\
\hline COL3A1 & X14420 & 18 & PSCA & AF043489 & 17 & $\begin{array}{l}\text { Ribosomal } \\
\text { protein } \\
\text { S29 }\end{array}$ & U14973 & 15 & $\begin{array}{l}\text { Cathepsin } \\
\text { B }\end{array}$ & L16510 & 9 \\
\hline $\begin{array}{l}\text { C04. Fibro- } \\
\text { blast* }^{*}\end{array}$ & 1097 & & $\begin{array}{l}\text { E05. Fundic } \\
\text { gland }\end{array}$ & 3304 & & $\begin{array}{l}\text { N10. } \\
\text { Fetal } \\
\text { neuron* }\end{array}$ & 1108 & & $\mathrm{X} 10$. Iris & 3314 & \\
\hline Stromelysin & X05232 & 26 & Pepsinogen & J00287 & 572 & $\begin{array}{l}\text { Ribosomal } \\
\text { protein } \\
\text { L37a }\end{array}$ & X66699 & 9 & $\begin{array}{l}\text { Apolipo- } \\
\text { protein } \\
\text { D }\end{array}$ & M16696 & 45 \\
\hline Fibronectin & K00799 & 22 & Lysozyme & $\times 14008$ & 33 & TPT-1 & X16064 & 9 & $1-8 D$ & $\times 57351$ & 43 \\
\hline Collagenase & X05231 & 15 & Gastric lipase & X05997 & 32 & $\begin{array}{l}\text { Ribosomal } \\
\text { protein } \\
\text { L5 }\end{array}$ & U14966 & 8 & $\begin{array}{l}\text { Yeast rp } \\
\text { L41 } \\
\text { homolog }\end{array}$ & Z12962 & 42 \\
\hline PAl-1 & $\times 13345$ & 14 & TPT-1 & $\times 16064$ & 29 & $\begin{array}{l}\text { Thymosin } \\
\text { B4 }\end{array}$ & M17733 & 8 & TPT-1 & $\times 16064$ & 40 \\
\hline $\begin{array}{l}\text { C05. } \\
\text { Mesangium }\end{array}$ & 1101 & & $\begin{array}{l}\text { E06. Ileum } \\
\text { epithel }\end{array}$ & 3675 & & $\begin{array}{l}\text { N11. } \\
\text { Corpus } \\
\text { callosum }\end{array}$ & 949 & & $\begin{array}{l}\text { X11. Skin } \\
\text { full } \\
\text { thickness }\end{array}$ & 4604 & \\
\hline Fibronectin & K00799 & 48 & $\begin{array}{c}\beta \text {-2-micro- } \\
\text { globulin }\end{array}$ & M17987 & 59 & GFAP & S40719 & 12 & $\begin{array}{l}\text { Yeast rp } \\
\text { L41 } \\
\text { homolog }\end{array}$ & Z12962 & 59 \\
\hline Calcyclin & J02763 & 30 & CLCA1 & AF039400 & 51 & $\begin{array}{c}\text { Ribosomal } \\
\text { protein } \\
\text { L37a }\end{array}$ & X66699 & 7 & GS20959 & None & 58 \\
\hline $\begin{array}{l}\text { ribosomal } \\
\text { protein } \$ 19\end{array}$ & M81757 & 13 & defensin 6 & M98331 & 46 & $\begin{array}{l}\text { ribosomal } \\
\text { protein } \\
\text { S8 }\end{array}$ & X67247 & 7 & $\begin{array}{l}\text { ribosomal } \\
\text { protein } \\
\text { S18 }\end{array}$ & X69150 & 57 \\
\hline
\end{tabular}


Table 1. (Continued)

\begin{tabular}{|c|c|c|c|c|c|c|c|c|c|c|c|}
\hline $\begin{array}{c}\text { Yeast rp S28 } \\
\text { homolog }\end{array}$ & D14530 & 10 & GS2706 & None & 34 & $\begin{array}{l}\text { Myelin basic } \\
\text { protein }\end{array}$ & M13577 & 7 & $\begin{array}{l}\text { Delta-6 } \\
\text { desaturase }\end{array}$ & AF03679 & 57 \\
\hline C06. Itoh cell & 1283 & & $\begin{array}{l}\text { E07. Colon } \\
\text { epithel }\end{array}$ & 6451 & & $\begin{array}{l}\text { N12. } \\
\text { Substantia } \\
\text { nigra }\end{array}$ & 3477 & & $\begin{array}{l}\text { X12. Tumor } \\
\text { infiltrates }\end{array}$ & 1585 & \\
\hline Osteonectin & J03040 & 23 & Galectin-4 & AF014838 & 106 & $\begin{array}{l}\text { Myelin basic } \\
\text { protein }\end{array}$ & M13577 & 129 & $\begin{array}{c}\beta \text {-2-micro- } \\
\text { globulin }\end{array}$ & M17987 & 49 \\
\hline Fibronectin & K00799 & 18 & cytokeratin 8 & X12882 & 86 & $\begin{array}{l}\text { Ribosomal } \\
\text { protein } \\
\text { L7a }\end{array}$ & X52138 & 51 & LD78 $\alpha$ & D90144 & 31 \\
\hline PAI-1 & X13345 & 16 & L-FABP & M10617 & 78 & $\mathrm{EF}-1 \alpha$ & X16869 & 48 & $\mathrm{RF}-1 \alpha$ & X16869 & 25 \\
\hline $\mathrm{EF}-1 \alpha$ & X16869 & 16 & Calcyclin & J02763 & 75 & $\alpha \mathrm{B}$-crystallin & S45630 & 46 & $\begin{array}{c}\text { Yeast rp S28 } \\
\text { homolog }\end{array}$ & D14530 & 24 \\
\hline $\begin{array}{l}\text { C07. Bone } \\
\text { flakes }\end{array}$ & 1042 & & $\begin{array}{l}\text { E08. } \\
\text { Pituitary }\end{array}$ & 1015 & & $\begin{array}{l}\text { N13. Fetal } \\
\text { brain }\end{array}$ & 3797 & & & & \\
\hline Osteonectin & J03040 & 25 & Prolactin & M29386 & 181 & $\alpha$-Tubulin & X01703 & 35 & & & \\
\hline COL1A2 & j03464 & 24 & $\begin{array}{l}\text { Growth } \\
\text { hormone }\end{array}$ & M13438 & 20 & $\begin{array}{l}\text { Ribosomal } \\
\text { protein } \\
\text { L37a }\end{array}$ & X66699 & 21 & & & \\
\hline$\beta$-Globin & V00497 & 13 & Secretogranin & Y00064 & 12 & $\mathrm{EF}-1 \alpha$ & X16869 & 20 & & & \\
\hline COL3A1 & X14420 & 12 & sGTP-bp & X07036 & 9 & Stathmin & J04991 & 16 & & & \\
\hline
\end{tabular}

The source tissue or cells and the number of total ESTs representing chromosomally encoded genes are given (shaded cells). The source groups were blood cells (B01-B06), connective and muscular tissues (C01-C12), epithelial tissues (E01-E08) and nervous tissues (N01-N13). When the source tissue was composed of multiple cell types or an uncategorizable cell type, it was categorized as complex (X01-X12). Asterisks denote primary cultured cells. The identities of the most frequently isolated tags are given along with their frequencies. (TPT-1) Translationally controlled tumor protein; (methionine AT-a) methionine adenosyltransferase- $\alpha$; (spermine AT) spermine acetyltransferase; (EF-1a) elongation factor 1- $\alpha$; (apM2) adipose most abundant protein-2; (PAI-1) plasminogen activator inhibitor-1; (ANF) atrial atriuretic factor; (EF-4AII) elongation factor 4All; (CLCA1) calcium activated chloride channel 1; (L-FABP) liver fatty acid binding protein; (sGTP-BP) stimulatory GTP bonding protein; (hng/RC3) human neurogranin; (GFAP) glial fibrillary acidic protein; (TALLA-1) T-cell acute lymphoblastic leukemia associated antigen 1; (pulmonary SAP) pulmonary surfactant apoprotein.

ing the $P$ value to $1 \%, 112$ more genes were selected (data not shown).

\section{DISCUSSION}

The wide coverage of human genes in dbEST permits parallel gene expression monitoring based on prior knowledge of gene sequence (Lockhart et al. 1996; Iyer et al. 1999). However, from a practical perspective, researchers must select a set of genes suitable for target tissues to make testing efficient (Loftus et al. 1999). The well-preserved abundance information and high anatomical resolution make BodyMap a preferable source for probe selection (http://bodymap.ims.u-tokyo.ac. jp). Another unique feature of BodyMap is the absolute abundance values for transcripts for various tissues. Such information is also found in shorter tag collection, SAGE (Velculescu et al. 1995; Welle et al. 1999), and the tissue-coverage complement to each other. The abundance data covering various tissues are complementary to relative gene expression comparison (DeRisi 1996; Schena et al. 1996; Kawamoto et al. 1999) for evaluating the functions of uncharacterized genes.

Site-directed EST sequences are indispensable for identification of gene ends within genomic sequences because even the most sophisticated computer pro- grams tend to overpredict the presence of exons (Dunham et al. 1999). The overlap of dbESTs with BodyMap indicates that there are still more transcripts to be identified in brain and other tissues. The higher complexity of transcripts in brain, as shown by the accumulated frequency curve, supports this idea. Possible overprediction of genes by using 3' ESTs is due to cloning artifacts and alternative polyadenylation. Validation of 3' ESTs by using hexanucleotide signals suggested that such artifacts were negligible in our data set. The $3^{\prime}$ ends without the AATAAA were observed at high incidences not only in BodyMap (39\%), but also in human cDNAs in GenBank (37\%) and qualified 3' ESTs from dbEST (40\%). In those 3 ' ends, several uncommon single-base variants, such BATAAA and AATABA $(B=T$, $G, C)$, plausibly responsible for poly(A) formation in these 3 ' ends, were found at very similar rates. After this paper was submitted, Beaudoing et al. (2000) published similar results from an analysis of 4344 human 3' untranslated regions (UTRs) and 3' ESTs overlapping with them. The proportion of 3 ' ends without AATAAA was $41.8 \%$ in their analysis, and uncommon singlebase variants were found at significant frequencies among them. In BodyMap, upstream alternative polyadenylation was found in $12 \%$ of GenBank mRNA entries. Assuming the same incidence of downstream alternatives, our estimate of alternative polyadenylation

\section{Genome Research}




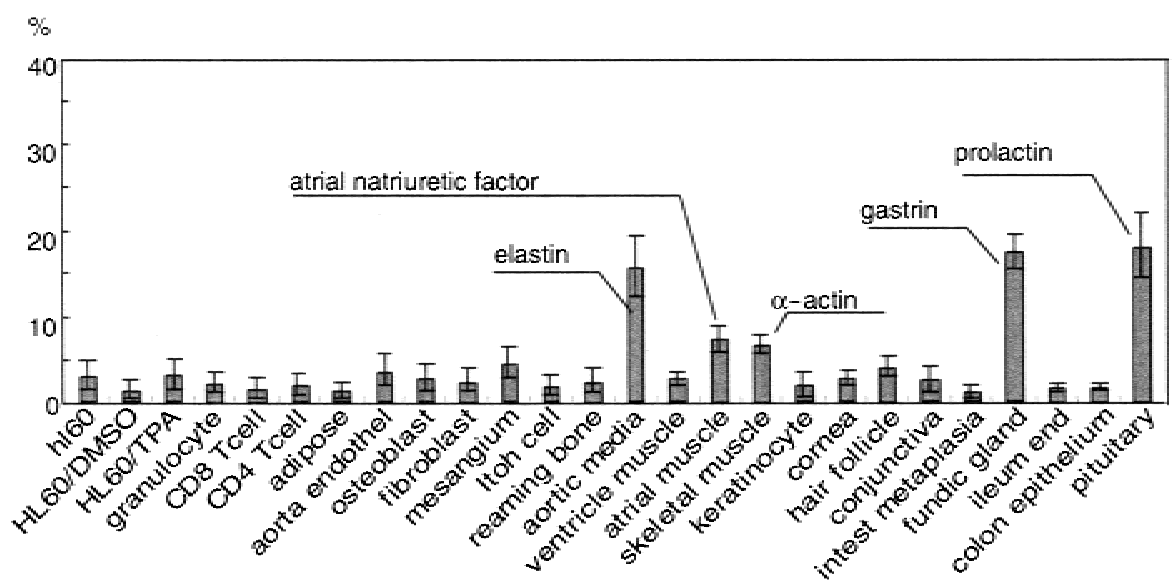

\section{METHODS}

\section{Library Construction}

Of 51 human libraries listed (Table 1), 15 were made by direct priming of total RNA. The specimens used for RNA preparations and the methods used are described elsewhere (http://bodymap.ims.utokyo.ac.jp). The other libraries were made from poly(A)-selected RNA. For counting transcripts by sequencing, only the most 3 'terminal fragment left by cleaving off the bulk of the fragment with $\mathrm{MboI}$ from the pUC119 vectorprimed cDNA was cloned, as described previously (Matsubara et al. 1993). This shortening of the inserts facilitates the unbiased representation of mRNA regardless of

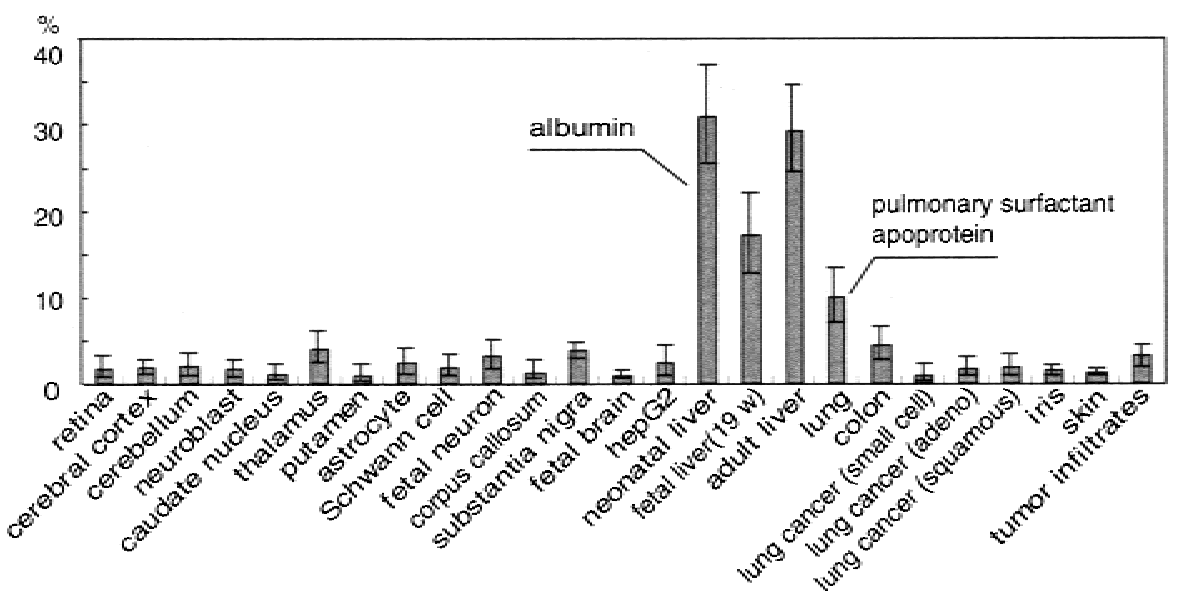

Figure 2 The relative contents of the most abundant transcripts in 51 human tissues or cell types as measured by gene signature collection. The error range indicates the $P$ value of 0.1 calculated for each observed occurrence. The identities of some transcripts are given. For the identities of other transcripts, see Table 1.

their original sizes at the expense of losing $\sim 5 \%$ of gene sequences due to the absence of $\mathrm{MboI}$ site or its location too close to the poly(A) tail.

\section{Data Collection and Cleansing}

Starting with randomly isolated transformants, sequence templates were prepared by PCR amplification of the insert cDNA in singlestranded phage released into the culture medium. All sequences were read from the Mbol site toward poly(A), which allows unambiguous identification of the original transcripts. They were referred to as GSs (Okubo et al. 1992). In half of the cases, dye primer chemistry was used, and in the remaining cases, DYEnamic ET* Terminator Cycle Sequencing Kit (Amer-

is $24 \%$, close to the reported estimates by EST clustering (16\%) (Gautheret et al.) and recent 3' UTR analysis (28.6\%). Although generation of multiple 3' ESTs from one gene may affect transcript counting by EST clustering, assigning them to genomic sequences will easily resolve this problem as long as the ESTs are not far apart.

In summary, our site-directed 3' ESTs can serve as a resource for selection of probes for sequence-based expression profiling methods and can provide absolute levels of gene expression that are important in considering gene function. Our collection covers various rare tissues and provides information on their mRNA populations. To allow full use of BodyMap for in silico mRNA experiments, the representation frequency matrix of gene $\times$ sources and all representative sequences have been made available through our ftp site (http:// bodymap.ims.u-tokyo.ac.jp/datasets/index.html). sham Pharmacia Biotech Inc.) was used. Sequences with $>5 \%$ Ns, not starting with GATC (the MboI site), or having more

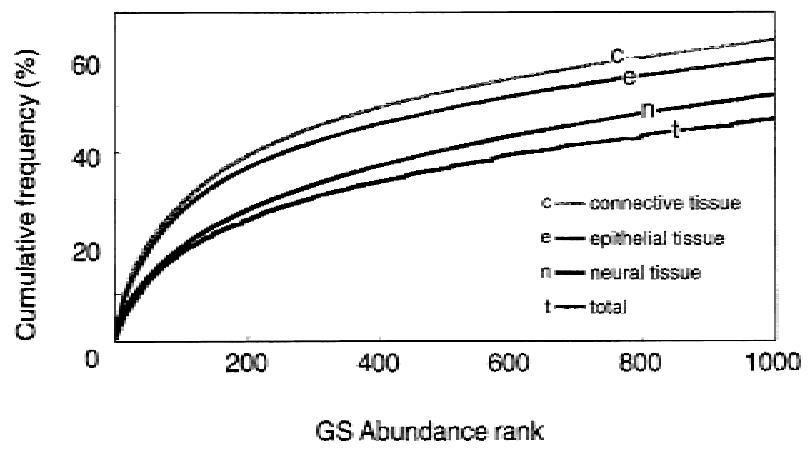

Figure 3 Cumulative frequencies of gene signature (GS) sequences. The cumulative sums calculated in descending order of GS frequencies are plotted as a percentage of total tag occurrence. Tag occurrences in each of three major tissue categories were plotted separately. 


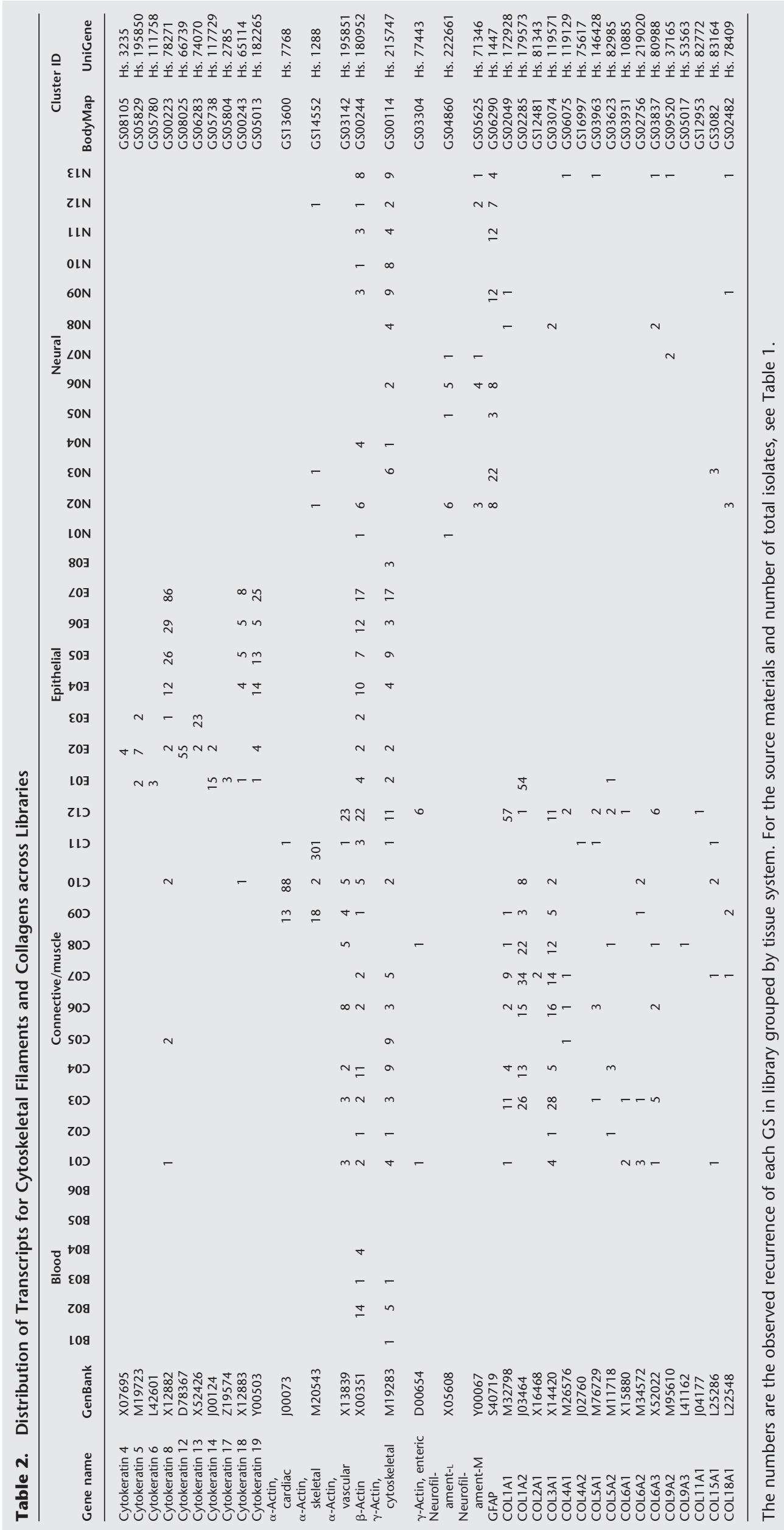


Table 3. Known Genes Selected as Uniquely Expressed in Myeloid Cells

\begin{tabular}{llll}
\hline Cluster ID & \multicolumn{1}{c}{ GenBank identity } & \#ACC & P value \\
\hline GS08362 & Granulocyte colony-stimulating factor receptor & S71484 & $2.56 \mathrm{E}-17$ \\
GS00697 & Plasminogen activator inhibitor-2 (PAI-2) & M24657 & $1.13 \mathrm{E}-09$ \\
GS01724 & Pleckstrin (P47) & X07743 & $1.13 \mathrm{E}-09$ \\
GS01024 & Leukocyte adhesion protein/CD18 & M15395 & $2.14 \mathrm{E}-08$ \\
GS01345 & Bactericidal permeability increasing protein & J04739 & $7.56 \mathrm{E}-06$ \\
& (BPI) & & \\
GS08325 & Phosphatidylinositol 3-kinase p110delta & U86453 & $7.56 \mathrm{E}-06$ \\
GS01990 & Secreted protein (I-309) & AF04502 & $7.56 \mathrm{E}-06$ \\
GS01200 & ICB-1 mRNA & M81750 & $1.42 \mathrm{E}-04$ \\
GS01719 & Myeloid cell nuclear differentiation antigen & $1.42 \mathrm{E}-04$ \\
GS01202 & Neutrophil oxidase factor (NCF2)/p67-phox & U00788 & $1.42 \mathrm{E}-04$ \\
GS00779 & Wegener's granulomatosis autoantigen & M97911 & $1.42 \mathrm{E}-04$ \\
& proteinase 3 & L00212 & $2.68 \mathrm{E}-03$ \\
GS01687 & C-raf-1 proto-oncogene & M60830 & $2.68 \mathrm{E}-03$ \\
GS01000 & EVI2B3P & M81637 & $2.68 \mathrm{E}-03$ \\
GS08337 & Grancalcin (neutrophil monocyte Ca binder) & U67615 & $2.68 \mathrm{E}-03$ \\
GS00610 & Beige protein homolog (chs) & M23197 & $2.68 \mathrm{E}-03$ \\
GS01164 & Differentiation antigen (CD33) & U47742 & $2.68 \mathrm{E}-03$ \\
GS08512 & Monocytic leukemia zinc finger protein & M21005 & $2.68 \mathrm{E}-03$ \\
GS01229 & Migration inhibitory factor-related protein 8 & AF057168 & $2.68 \mathrm{E}-03$ \\
GS01963 & Type II interleukin-1 receptor antagonist & & \\
& (IL-1ra3) & &
\end{tabular}

Gene signature (GS) clusters were selected by the criteria that have probability of uncontrolled expression between myeloid cells (HL60, HL60/DMSO, HL60/TPA, granulocytes) and the remaining tissues less than $0.5 \%$. $P$ values represent the probabilities of each gene with uncontrolled expression between two sets of libraries (see Methods for calculations). Along with these known genes, $28 \mathrm{GSs}$ for novel genes as follows were selected: GS01371, GS01572, GS08424, GS01582, GS01553, GS01965, GS01356, GS00656, GS08595, GS01922, GS08435, GS01123, GS00963, GS01383, GS08551, GS08572, GS01352, GS01109, GS01561, GS08460, GS05157, GS00549, GS01251, GS00627, GS08477, GS08379, GS01458, GS01987. For sequences, refer to http://bodymap.ims.u-tokyo.ac.jp.

than one GATC were eliminated. We then eliminated those sequences having $>90 \%$ similarity in an overlap longer than $50 \mathrm{bp}$ or $70 \%$ of the sequence length with vectors and ribosomal sequences. Sequences for mitochondrial transcripts were also eliminated. When the GATC and poly(A) tail were separated by $<17 \mathrm{bp}$, the sequences were eliminated from the analysis because they were not always unique enough. Lastly, sequences were compared with a library of repetitive sequences, REPBASE (Jurka 1995, ftp://ncbi.nlm.nih.gov/ repository/repbase/) by using BLAST (Altschul et al. 1990), and repetitive regions were masked as previously reported (Hishiki et al. 2000). All GS sequences were submitted to the DNA DataBank of Japan (DDBJ) and made available at our web site (http://bodymap.ims.u-tokyo.ac.up).

\section{Transcript Counting/EST Clustering}

Sequences from each new library were first compared to each other with FASTA (Pearson et al. 1988). When the similarity exceeded $95 \%$ for an overlap longer than 50 bp or $70 \%$ of insert length and the overlap started at a GATC, they were considered the same tag and clustered (primary cluster). From each cluster, one representative GS was selected and compared with representative sequences from previously generated clusters. By using the same criteria, clusters of the same GS were grouped, and a new representative tag was selected from the new cluster (secondary cluster). A five-figure cluster ID, referred to as the GS number, was assigned to each independent cluster. Representative sequences for the GS clusters were compared periodically with primate sequences in Gen-
Bank (Re. 110.0) and ESTs in UniGene (Build 75, http:// www.ncbi.nlm.nih.gov/UniGene/). The criteria for identity were the same as those used for clustering. The correspondence of BodyMap ID (GS) to UniGene ID (Hs) was submitted to GenBank and implemented in UniGene.

\section{Selection of Differentially Expressed Genes}

For the selection of genes preferentially expressed in a given set of tissues, for example tissues $\mathrm{A}, \mathrm{B}$, and $\mathrm{C}$, libraries $\mathrm{A}-\mathrm{C}$ were considered one library and the remaining 48 libraries in BodyMap another library. The probability of unregulated expression between the two hypothetical libraries was calculated for each GS by the equation reported by Audic and Claverie (Audic et al. 1997):

$$
P(y \mid x)=\left(\frac{N 2}{N 1}\right)^{y} \frac{(x+y) !}{x ! y !\left(1+\frac{N 2}{N 1}\right)^{(x+y+1)}}
$$

Total isolation in A-C is $\mathrm{N} 1$ and isolation of the relevant GS is $x$. The total isolation in the remaining libraries is $N 2$ and the occurrence of the relevant GS is $y$.

\section{Analysis of Polyadenylation Signals}

Among 62,710 entries of primate sequences in GenBank (Re.97), all human mRNAs with a single "poly(A)-site" listed in the features were used. From the representative sequences for all GSs, we selected those that satisfied all of the following conditions. The GS does not have matches in GenBank, is 
longer than $100 \mathrm{bp}$, and ends with poly(A). The GS sequence does not contain more than 5\% Ns within $100 \mathrm{bp}$ of the poly(A). The GS does not contain repetitive sequences or an $\mathrm{N}$ in the AATAAA sequence, such as 'NATAAA'.

From dbEST (Re. 93), we selected 118,353 3' ESTs from the Washington-U/Merck project (Hillier et al. 1996) to avoid confusion due to inconsistencies in the feature descriptions from different laboratories. EST matches to BodyMap entries and GenBank primate mRNAs were eliminated first. Those ESTs with discrepancies between clone name and definition ( $5^{\prime}$ in clone name and $3^{\prime}$ EST in definition), and those denoted as "possible reverse clone" were also eliminated. $3^{\prime}$ ESTs with a stretch of longer than seven Ts $(\mathrm{Tn}>7)$ at the beginning and those starting with $\mathrm{A}, \mathrm{G}$, or $\mathrm{C}\left(\mathrm{T}_{0}\right)$ were analyzed separately. Those ESTs starting with one to seven Ts were not used. Within each of these four categories, the 100 bases from the poly(A) site were compared with each other with BLAST N with the same criteria used for GS clustering, and the fragment containing the lowest number of Ns was selected from each cluster and used in the analysis.

\section{ACKNOWLEDGMENTS}

The authors thank Ms. Kumiko Takagi for her secretarial assistance. This work was supported in part by Grant-in-Aid for Scientific Research on Priority Areas from the Ministry of Education, Science and Culture, and Research for the future of Japan Society for the Promotion of Science, Japan.

The publication costs of this article were defrayed in part by payment of page charges. This article must therefore be hereby marked "advertisement" in accordance with 18 USC section 1734 solely to indicate this fact.

\section{REFERENCES}

Aaronson, J.S., Eckman, B., Blevins, R.A., Borkowski, J.A., Myerson, J., Imran, S., and Elliston, K.O. 1996. Toward the development of a gene index to the human genome: An assessment of the nature of high-throughput EST sequence data. Genome Res. 6: 829-845.

Adams, M.D., Kerlavage, A.R., Fields, C., and Venter, J.C. 1993. 3,400 new expressed sequence tags identify diversity of transcripts in human brain. Nat. Genet. 4: 256-267.

Adams, M.D., Kerlavage, A.R., Fleischmann, R.D., Fuldner, R.A., Bult, C.J., Lee, N.H., Kirkness, E.F., Weinstock, K.G., Gocayne, J.D., White, O., et al. 1995. Initial assessment of human gene diversity and expression patterns based upon 83 million nucleotides of cDNA sequence. Nature 377: 3-174.

Altschul, S.F., Gish, W., Miller, W., Myers, E.W., and Lipman, D.J. 1990. Basic local alignment search tool. J. Mol. Biol. 215: 403-410.

Audic, S. and Claverie, J.-M. 1997. The significance of digital gene expression profiles. Genome Res. 7: 986-995.

Beaudoing, E., Freier, S., Wyatt, J.R., Claverie, J-M., and Gautheret, D. 2000. Patterns of variant polyadenylation signal usage in human genes. Genome Res. 10: 1001-1010.

Bonaldo, M.F., Lennon, G., and Soares, M.B. 1996. Normalization and subtraction: Two approaches to facilitate gene discovery. Genome Res. 6: 791-806.

DeRisi, J., Penland, L., Brown, P.O., Bittner, M.L., Meltzer, P.S., Ray, M., Chen, Y., Su, Y.A., and Trent, J.M. 1996. Use of a cDNA microarray to analyse gene expression patterns in human cancer. Nat. Genet. 14: 457-460.

Dunham, I., Shimizu, N., Roe, B.A., Chissoe, S., Hunt, A.R., Collins, J.E., Bruskiewich, R., Beare, D.M., Clamp, M., Smink, L.J., et al. 1999. The DNA sequence of human chromosome 22. Nature 402: 489-495.

Gautheret, D., Poirot, O., Lopez, F., Audic, S., and Claverie, J.-M. 1998. Alternate polyadenylation in human mRNAs: A large-scale analysis by EST clustering. Genome Res. 8: 524-530.
Hattori, M., Fujiyama, A., Taylor, T.D., Watanabe, H., Yada, T., Park, H.S., Toyoda, A., Ishii, K., Totoki, Y., Choi, D.K., et al. 2000. The DNA sequence of human chromosome 21. Nature 405: 311-319.

Hillier, L.D., Lennon, G., Becker, M., Bonaldo, M.F., Chiapelli, B., Chissoe, S., Dietrich, N., DuBuque, T., Favello, A., Gish, et al. 1996. Generation and analysis of 280,000 human expressed sequence tags. Genome Res. 6: 807-828.

Hishiki, T., Kawamoto, S., Morishita, S., and Okubo, K. 2000. BodyMap: A human and mouse gene expression database. Nucleic Acids Res. 28: 136-138.

Irvine, A.D., Corden, L.D., Swensson, O., Swensson, B., Moore, J.E., Frazer, D.G., Smith, F.J., Knowlton, R.G., Christophers, E., Rochels, R., et al. 1997. Mutations in cornea-specific keratin K3 or K12 genes cause Meesmann's corneal dystrophy. Nat. Genet. 16: $184-187$.

Iyer, V.R., Eisen, M.B., Ross, D.T., Schuler, G., Moore, T., Lee, J.C.F., Trent, J.M., Staudt, L.M., Hudson, J., Jr., Boguski, M.S., et al. 1999. The transcriptional program in the response of human fibroblasts to serum. Science 283: 83-87.

Kawamoto, S., Ohnishi, T., Kita, H., Chisaka, O., and Okubo, K. 1999. Expression profiling by iAFLP: A PCR-based method for genome-wide gene expression profiling. Genome Res. 9: $1305-1312$.

Lockhart, D.J., Dong, H., Byrne, M.C., Follettie, M.T., Gallo, M.V., Chee, M.S., Mittmann, M., Wang, C., Kobayashi, M., Horton, H., et al. 1996. Expression monitoring by hybridization to high-density oligonucleotide arrays. Nat. Biotechnol. 14: $1675-1680$

Loftus, S.K., Chen, Y., Gooden, G., Ryan, J.F., Birznieks, G., Hilliard, M., Baxevanis, A.D., Bittner, M., Meltzer, P., Trent, J., et al. 1999. Informatic selection of a neural crest-melanocyte cDNA set for microarray analysis. Proc. Natl. Acad. Sci. 96: 9277-9280.

Maeda, K., Okubo, K., Shimomura, I., Mizuno, K., Matsuzawa, Y., and Matsubara, K.1997. Analysis of an expression profile of

Matsubara, K, and Okubo, K. 1993. cDNA analyses in the human genome project. Gene 135: 265-274.

Nishida, K., Adachi, W., Shimizu-Matsumoto, A., Kinoshita, S., Mizuno, K., Matsubara, K., and Okubo, K. 1996. A gene expression profile of human corneal epithelium and the isolation of human keratin 12 cDNA. Invest. Ophthalmol. Vis. Sci. 37: 1800-1809.

Nishida, K., Honma, Y., Dota, A., Kawasaki, S., Adachi, W., Nakamura, T., Quantock, A. J., Hosotani, H., Yamamoto, S., Okada, M.,et al. 1997. Isolation and chromosomal localization of a cornea-specific human keratin 12 gene and detection of four mutations in Meesmann corneal epithelial dystrophy. Am. J. Hum. Genet. 61: 1268-1275.

Ohnishi, T. and Okubo, K. 1999. Isolation of pure human mucosal epithelium for RNA analysis. Biotechniques 27: 978- 986.

Ohno, I., Hashimoto, J., Shimizu, K., Takaoka, K., Ochi, T., Matsubara, K., and Okubo, K. 1996. A cDNA cloning of human AEBP1 from primary cultured osteoblasts and its expression in a differentiating osteoblastic cell line. Biochem. Biophys. Res. Commun. 228: 411-414.

Okubo, K., Hori, N., Matoba, R., Niiyama, T., and Matsubara, K. 1991. A novel system for large-scale sequencing of cDNA by PCR amplification. DNA Seq. 2: 137-144.

Okubo, K., Hori, N., Matoba, R., Niiyama, T., Fukushima, A., Kojima, Y., and Matsubara, K. 1992. Large scale cDNA sequencing for analysis of quantitative and qualitative aspects of gene expression. Nat. Genet. 2: 173-179.

Pearson, W.R. and Lipman, D.J. 1988. Improved tools for biological sequence comparison. Proc. Natl. Acad. Sci. 85: 2444-2448.

Schena, M., Shalon, D., Heller, R., Chai, A., Brown, P.O., and Davis, R.W. 1996. Parallel human genome analysis: Microarray-based expression monitoring of 1000 genes. Proc. Natl. Acad. Sci. 93: 10614-10619.

Schuler, G.D., Boguski, M.S., Stewart, E.A., Stein, L.D., Gyapay, G., Rice, K., White, R.E., Rodriguez-Tome, P., Aggarwal, A., Bajorek, E., et al. 1996. A gene map of the human genome. Science 274: $540-546$. 
Sheets, M.D., Ogg, S.C., and Wickens, M.P. 1990. Point mutations in AAUAAA and the poly (A) addition site: Effects on the accuracy and efficiency of cleavage and polyadenylation in vitro. Nucleic Acids Res. 18: 5799-5805.

Shimizu-Matsumoto, A., Adachi, W., Mizuno, K., Inazawa, J., Nishida, K., Kinoshita, S., Matsubara, K., and Okubo, K. 1997. An expression profile of genes in human retina and isolation of a complementary DNA for a novel rod photoreceptor protein. Invest. Ophthalmol. Vis. Sci. 38: 2576-2585.

Soares, M.B., Bonaldo, M.F., Jelene, P., Su, L., Lawton, L., and Efstratiadis, A. 1994. Construction and characterization of a normalized cDNA library. Proc. Natl. Acad. Sci. 91: 9228-9232.

Strausberg, R.L., Buetow, K.H., Emmert-Buck, M.R., and Klausner, R.D. 2000. The cancer genome anatomy project: Building an annotated gene index. Trends Genet. 16: 103-106.

Velculescu, V.E., Zhang, L., Vogelstein, B., and Kinzler, K.W. 1995. Serial analysis of gene expression. Science 270: $484-487$.

Welle, S., Bhatt, K., and Thornton, C.A. 1999. Inventory of high-abundance mRNAs in skeletal muscle of normal men. Genome Res. 9: 506-513.

Wickens, M. and Stephenson, P. 1984. Role of the conserved AAUAAA sequence: Four AAUAAA point mutants prevent messenger RNA 3' end formation. Science 226: 1045-1051.

Williamson, A. R. 1999. The Merck Gene Index project. Drug Discov. Today 4: 115-122.

Received June 8, 2000; accepted in revised form September 18, 2000. 


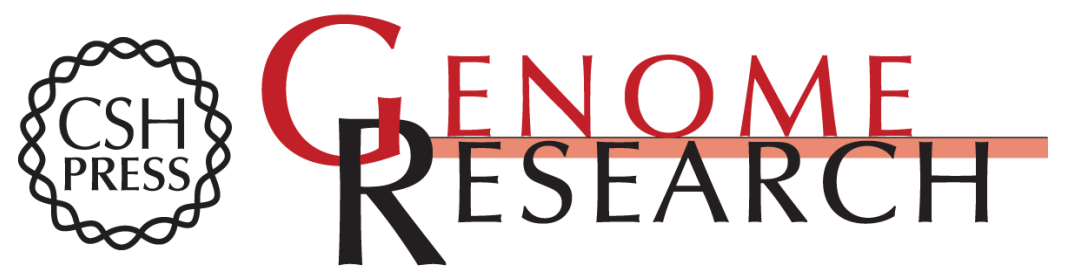

\section{BodyMap: A Collection of 3' ESTs for Analysis of Human Gene Expression Information}

Shoko Kawamoto, Junji Yoshii, Katsuya Mizuno, et al.

Genome Res. 2000 10: 1817-1827

Access the most recent version at doi:10.1101/gr.151500

References This article cites 36 articles, 18 of which can be accessed free at:

http://genome.cshlp.org/content/10/11/1817.full.html\#ref-list-1

\section{License}

Email Alerting Receive free email alerts when new articles cite this article - sign up in the box at the Service top right corner of the article or click here.

\section{Affordable, Accurate Sequencing.}

To subscribe to Genome Research go to: https://genome.cshlp.org/subscriptions 